\title{
AVALIAÇÃO SENSORIAL DE CACHAÇA
}

\section{Luigi Odello e Gian Paolo Braceschi}

Centro Studi Assaggiatori, Galleria V.Veneto 9, 25128, Brescia, Itália

Fernanda Rosan Fortunato Seixas, Alexandre Ataide da Silva, Carlos Alexandre Galinaro e Douglas Wagner Franco* Instituto de Química de São Carlos, Universidade de São Paulo, Av. do Trabalhador Sancarlense, 400, 13560-970 São Carlos - SP, Brasil

Recebido em 23/10/08; aceito em 16/3/09; publicado na web em 31/8/09

\begin{abstract}
SENSORY EVALUATION OF CACHAÇA. The hedonic level of commercial cachaças, was evaluated by consumers and by a tasters. The results of sensorial methods analyzed trough Principal Components Analysis, Hierarchical Cluster Analysis and the Pearson linear correlation indicated that the best classified cachaças were produced in copper stills and aged in oak casks. By contrast the worst classified exhibited as the main features be not aged and high alcohol percentage. The index of preference is positively correlated with the intensity of yellow color, wood flavor, sweetness and fruit aroma. There is a negative preference correlation with the acidity, the taste of alcohol and bitterness.
\end{abstract}

Keywords: cachaça; sensory analysis; hedonic level.

\section{INTRODUÇÃO}

A cachaça, destilado do vinho obtido a partir do mosto fermentado de cana-de-açúcar, gerou uma receita de US\$ 14,4 milhões em 2007. ${ }^{1}$ Este valor tende a aumentar em virtude dos esforços para a sua comercialização no exterior e da denominação de origem que classificou a cachaça como sendo um produto típico do Brasil pelo Decreto $n^{\circ} .4062$ de 21/12/2001. ${ }^{2}$

Esta bebida é constituída majoritariamente por etanol e água, e também por compostos secundários tais como álcoois superiores, ${ }^{3}$ ácidos, ${ }^{4,5}$ ésteres, ${ }^{6}$ acetais,${ }^{7}$ fenóis, ${ }^{8}$ hidrocarbonetos, ${ }^{9}$ compostos nitrogenados ${ }^{10}$ sulfurados ${ }^{11}$ e açúcares, entre outros, que a caracterizam e a qualificam. ${ }^{12,13}$

A cachaça é muito apreciada por seu sabor e aroma característicos, que são decorrentes dos processos de fermentação, destilação e envelhecimento em tonéis de madeira, sendo denominada cachaça envelhecida a bebida que contiver no mínimo $50 \%$ de aguardente de cana envelhecida em barris de madeira, por um período não inferior a 1 ano, podendo ser adicionada de caramelo para a correção da cor. ${ }^{14}$

Claramente impulsionado pela necessidade de conquista do mercado externo, existe um esforço do setor produtivo e dos laboratórios de pesquisa para a melhoria da qualidade da cachaça. A descrição qualitativa e quantitativa dos compostos químicos presentes em cachaça tem recebido constante atenção por parte de diversos Laboratórios. No entanto, a caracterização da cachaça somente sobre o ponto de vista químico, apesar de extremamente relevante, não é suficiente, necessitando ser complementada pelo conhecimento dos atributos sensoriais da bebida.

A definição das substâncias de impacto sensorial que compõem uma bebida destilada é fundamental no monitoramento da produção, na modificação de suas características e para o controle de sua qualidade. A correlação entre os componentes responsáveis pelo aroma, sabor e aspecto visual com a qualidade da bebida são objetos da análise sensorial. Essa continua sendo a principal forma de avaliar a aceitação das mesmas pela percepção humana. ${ }^{15}$

*e-mail: douglas@iqsc.usp.br
O presente trabalho reporta pela primeira vez o índice hedônico e de preferência da aguardente de cana brasileira, certificadas e um número significativo de amostras e de avaliadores.

\section{PARTE EXPERIMENTAL}

\section{Material}

Foram analisadas quimicamente 36 amostras de cachaças comerciais, não adoçadas. Considerando os padrões estabelecidos pela Instrução Normativa do Ministério da Agricultura, Pecuária e Abastecimento, ${ }^{14}$ foram aprovadas para a avaliação sensorial apenas 20 cachaças, sendo 8 não envelhecidas e 12 envelhecidas: Cachaça Engenho da Vertente (SP); Cachaça Uma (SP); Reserva do Tanoeiro (SP); Cachaça do Porão (SP); Cachaça Panorama (SP); Cachaça Três Garças (SP); Cachaça Santa Inês (SP); Aguardente Tiquara (SP); Cachaça Capilé (SP); Cachaça Monções (SP); Cachaça Gabriela (SP); Aguardente São Saruê (PE); Cachaça Aroma do Caraça (MG); Caninha Tietê (SP); Cachaça Campanari (SP); Cachaça do Rei Premium (SP); Cachaça do Rei Gold (SP); Cachaça Volúpia Envelhecida (PB); Cachaça Vale das Águas Quentes Sênior (GO); Cachaça Jequity Branca (SP).

\section{Parâmetros tecnológicos das amostras de cachaça}

Foram solicitadas, aos produtores, as seguintes informações sobre os processos utilizados na produção das cachaças: tipo de alambique em que as amostras foram destiladas, tipo de coluna, se as mesmas foram envelhecidas ou não, qual o tipo da madeira do tonel utilizado no envelhecimento e o tempo de envelhecimento (meses).

\section{Análise química}

Foram realizadas as seguintes análises químicas: teor alcoólico, ${ }^{16}$ cobre/ferro, ${ }^{8}$ carbamato de etila, ${ }^{17}$ dimetilsulfeto (DMS ${ }^{18}$ e caramelo. ${ }^{19}$

\section{Teor alcoólico}

O teor alcoólico foi determinado por densitometria a $20{ }^{\circ} \mathrm{C}$, utilizando-se o método do picnômetro. ${ }^{16}$ 


\section{Carbamato de etila e dimetilsulfeto}

Para estas análises foi utilizado um cromatógrafo a gás equipado com um detector de massas Shimadzu (GCMS-5050A) operando no modo SIM monitorando o íon $\mathrm{m} / \mathrm{z}=62$. A determinação do dimetilsulfeto foi realizada utilizando-se um concentrador purge and trap (OI Analytical, modelo 4560) acoplado ao cromatógrafo. ${ }^{18}$

\section{Cobre e ferro}

As análises de cobre e ferro foram realizadas por espectrofotometria de absorção atômica utilizando-se um espectrofotômetro Hitachi Z-8100 com chama de ar-acetileno. ${ }^{8}$

\section{Caramelo}

A análise de caramelo foi efetuada utilizando-se um espectrofotômetro UV-visível (HP - Hewlett Packard, 8452 A), segundo metodologia citada anteriormente. ${ }^{19}$

\section{Análise sensorial}

Para a realização da avaliação sensorial e hedônica foram utilizados dois níveis diferentes de avaliação: avaliação sensorial de descritores quantitativos e qualitativos por parte de um painel de especialistas treinados e a avaliação da preferência global por parte de consumidores. Os testes foram realizados em salas apropriadas do Instituto de Química de São Carlos da Universidade de São Paulo (IQSC-USP) seguindo as normas da Associação Brasileira de Normas Técnicas. ${ }^{20}$ As amostras foram codificadas, apresentadas de forma equitativa, em temperatura ambiente $\left(25^{\circ} \mathrm{C}\right)$ e em taças transparentes tipo ISO.

\section{Método sensorial descritivo}

Esta técnica foi utilizada para identificar e quantificar a intensidade dos atributos sensoriais do produto ${ }^{21}$ por meio de uma equipe (painel) composta inicialmente de 24 provadores de ambos os sexos, selecionados e treinados por um líder. O grupo era constituído de técnicos, operários, produtores e estudantes. Foram avaliadas a atitude dos participantes no uso dos órgãos do sentido como instrumento de análise sensorial e a sua capacidade de julgamento, com o propósito de treinar o painel para sucessivas análises das amostras.

Para a avaliação das amostras utilizou-se o método Trialtest $t^{21}$ que permitiu identificar o perfil descritivo do produto e considerar somente 13 juízes confiáveis. Os juízes foram considerados confiáveis com base no poder de discriminação entre amostras, repetibilidade e concordância entre os provadores. Posteriormente, as avaliações foram comparadas estatisticamente pelo teste de diferença mínima significativa não paramétrico (least significant difference - LSD). No primeiro teste obteve-se a extração das descrições pelo painel sobre as amostras (Perfil Descritivo Semântico-PDS), ${ }^{22}$ para utilizá-las nas fases de avaliação. Sucessivamente foi realizada entre os juízes e o líder de painel uma discussão (mesa redonda) para reagrupar as descrições com o mesmo significado e eliminar as descrições não percebidas por um número significativo dos juízes. Desta forma, definiu-se o perfil descritivo do produto a ser utilizado em seguida para a avaliação das amostras. Esta foi realizada segundo as características da análise sensorial partindo da avaliação visiva, olfativa, gustativa e retrolfativa. ${ }^{22,23} \mathrm{O}$ perfil quantitativo foi resultado da elaboração, por meio da mediana, dos dados relativos à avaliação por parte dos 13 juízes, quanto a oleosidade, intensidade de cor amarela, calor, ardor, doçura, acidez, maciez/suavidade, amargor e os sabores floral, frutado, madeira e álcool.

\section{Método sensorial afetivo}

Esse método avaliou a preferência dos consumidores pelo produto, segundo a metodologia descrita por Stratus tasting. ${ }^{23}$ Para tanto foi utilizada uma ficha estruturada para a determinação do índice de preferência das amostras de cachaça, a qual solicitava ao consumidor retratar seu juízo sobre o produto degustado. As sensações avaliadas foram: visiva, olfativa, gustativa e gustolfativa. Os consumidores atribuíram para cada percepção um valor de 1 a 6 . A escolha desta escala teve como objetivo minimizar a atribuição por parte do avaliador de um valor central da escala. Para cada consumidor foram servidos cinco produtos não identificados, de modo a assegurar os mesmos números de degustações para todas as amostras. Foram geradas séries casuais de amostras. Os participantes eram principalmente do sexo masculino (77\%) com idade entre 18 a 50 anos, basicamente estudantes, produtores de cachaça e funcionários do IQSC-USP.

\section{Análise quimiométrica}

Os resultados foram avaliados utilizando o programa estatístico Big Sensory Soft $t^{\circledR} 1.0$ versão 2005. ${ }^{24}$ Para a análise de componentes principais (PCA, Principal Components Analysis) e análise hierárquica de agrupamento (HCA, Hierarchical Cluster Analysis) utilizou-se o programa MINITAB Statistical Software ${ }^{\circledR}$ versão $14 .{ }^{25} \mathrm{Na} P C A$ os dados foram projetados através de combinações lineares das variáveis originais (oleosidade, intensidade de cor amarela, calor, ardor, doçura, acidez, maciez/suavidade, amargor e os sabores floral, frutado, madeira e álcool), formando as componentes principais (PC). Foram então obtidos os gráficos de scores e de loading, isto é, a distribuição das amostras em função da importância das variáveis.

A $H C A$, baseada nos valores da mediana de cada atributo avaliado na análise sensorial por parte dos especialistas, foi usada com o objetivo de verificar as semelhanças relativas entre as amostras(distância Euclidiana). Os resultados da $H C A$ foram representados na forma de um gráfico bidimensional (dendrograma).

O gerador hedônico foi desenvolvido utilizando a correlação entre descrições objetivas de análise sensorial e de índice global de preferência dos consumidores (coeficiente de correlação linear de Pearson) ${ }^{23}$ Os resultados foram apresentados de forma tabular e gráfica.

\section{RESULTADOS E DISCUSSÃO}

Segundo os processos tecnológicos utilizados na produção das cachaças (Tabela 1S, Material Suplementar), a maioria das amostras foi destilada em alambique de cobre (95\%), e envelhecida em barris de carvalho $(66 \%)$.

Os resultados das análises químicas (Tabela 2S, Material Suplementar) demonstraram uma variação no teor alcoólico de 30,0 a $44,0 \%$. Para o dimetilsulfeto estes teores variaram de não detectado a $8,91 \mathrm{mg} \mathrm{L}^{-1}$, para o cobre de $4,60 \times 10^{-2}$ a $1,10 \mathrm{mg} \mathrm{L}^{-1}$, para carbamato de etila de $3,20 \times 10^{-2}$ a $1,30 \times 10^{-1} \mathrm{mg} \mathrm{L}^{-1}$, para o ferro de $8,00 \times 10^{-4}$ a 7,10 x $10^{-2} \mathrm{mg} \mathrm{L}^{-1}$, para o caramelo de não detectado a $3,40 \mathrm{~g} \mathrm{~L}^{-1}$.

$\mathrm{O}$ índice hedônico das amostras analisadas pelo painel de especialistas apresentou diferenças significativas (Figura 1S, Material Suplementar), sendo preferidas as amostras 19, 24, 5, 33 e 12, com valores superiores a 6,0. O perfil quantitativo dessas amostras (exceto a 12) pode ser observado na Figura 1. A utilização do gráfico radar para demonstração do perfil sensorial nos permite uma melhor visualização dos itens de impacto, positivo e negativo. ${ }^{26}$

O índice hedônico é dado pelo balanço harmônico entre as características positivas e negativas da cachaça. A amostra 19 (Tabela 3S, Material Suplementar) foi a melhor classificada, destacando-se por apresentar os menores valores com respeito a calor, ardor, amargor, 
Amostra 19

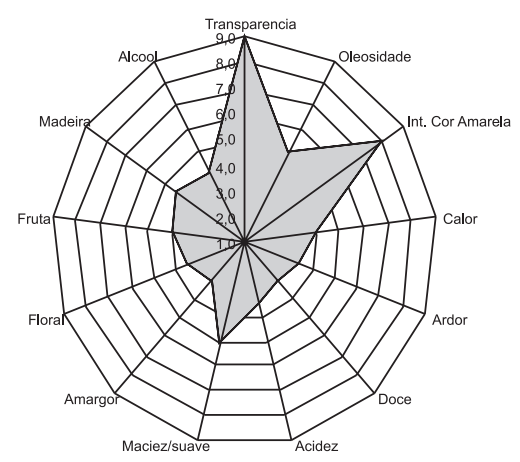

Amostra 24

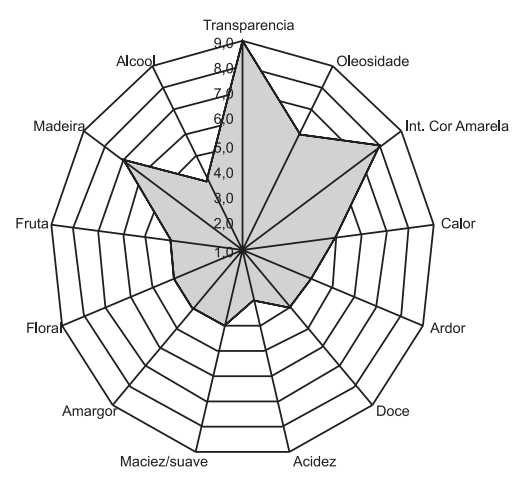

Amostra 5

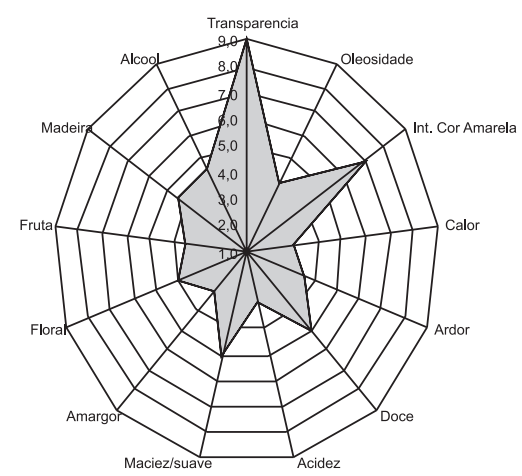

Amostra 33

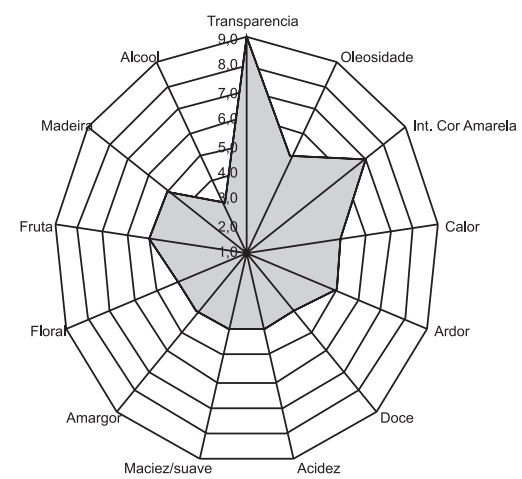

Figura 1. Perfil quantitativo (gráfico radar) das melhores cachaças por parte da avaliação do painel de especialistas (exceto amostra 12)

considerados indicadores negativos da qualidade da cachaça, e os maiores valores de intensidade de cor amarela e para a relação maciez/ suavidade que são indicadores positivos.

As amostras melhores qualificadas foram destiladas em alambique de cobre. As cachaças destiladas em alambique de cobre apresentam um teor médio total de álcoois superiores inferior aos das destiladas em coluna de aço inox. ${ }^{27}$ Além de sua participação na formação de aldeídos, o cobre metálico presente nos alambiques age de forma a reduzir o teor de compostos sulfurados voláteis no destilado ${ }^{28}$ e, portanto, o desagradável odor típico de sulfetos.

As amostras melhores classificadas foram as envelhecidas em barris de carvalho (Quercus sp.). As amostras 19 e 33 foram envelhecidas por um período de 48 meses e as amostras $24,5,12$ por 24 meses. Uma correlação entre o nível hedônico e o tipo de envelhecimento pode ser observada na Figura 2. Essa observação é compatível com o fato das características sensoriais de cachaças envelhecidas serem dependentes da somatória de congêneres e dos extrativos da madeira ${ }^{26,29}$ ao longo do tempo de envelhecimento. Sabe-se que os principais compostos extraídos da madeira do tonel pelos destilados são: óleos voláteis, substâncias tânicas, açúcares, glicerol, ácidos orgânicos não voláteis, esteróides, os quais modificam o aroma, sabor e a coloração da bebida. ${ }^{30}$

Assim, a prática do envelhecimento promove diminuição significativa do sabor alcoólico e da agressividade, com simultâneo aumento da doçura e do sabor de madeira, proporcionando uma efetiva melhora sensorial do produto. ${ }^{12}$

Na Figura 2 observa-se semelhança entre o índice hedônico das cachaças não envelhecidas e das envelhecidas em jequitibá (Cariniana estrellensis), considerada uma madeira neutra. A amostra 20 foi envelhecida em jequitibá por período de 36 meses e, como esperado, apresentou nível hedônico superior ao das cachaças não envelhecidas e próximo aos das envelhecidas em carvalho por período de 24 meses.

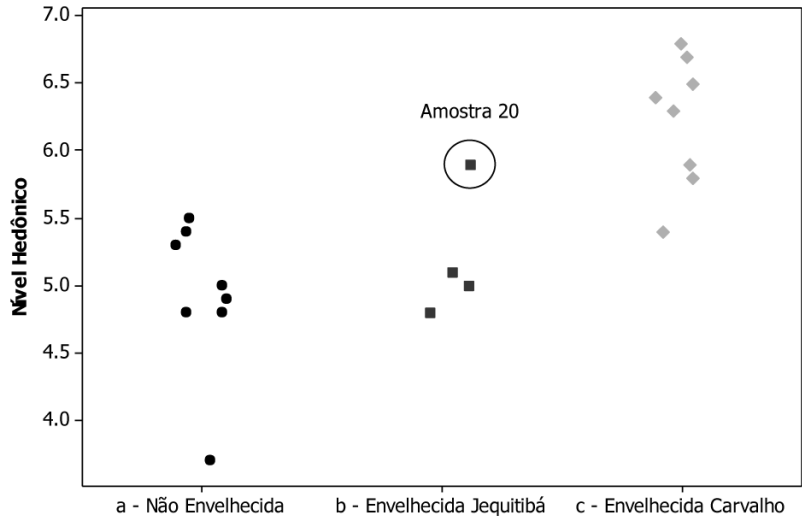

Figura 2. Relação entre nível hedônico (painel de especialistas) e o processo de envelhecimento

As amostras 23, 8 e 7 foram as piores classificadas na preferência do painel de juízes qualificados (Figura 1S, Material Suplementar), apresentando características diferentes com relação ao seu processo de produção. A amostra 23 não foi envelhecida, apenas descansada ou repousada, ou seja, armazenada por um período de até 6 meses em barris de madeira. O perfil sensorial quantitativo dessas amostras (exceto a 7) pode ser observado na Figura 3. Estas duas amostras foram consideradas semelhantes principalmente pelos elevados valores atribuídos às sensações de calor e ardor, que se correlacionam negativamente com o índice hedônico (Tabela 3S, Material Suplementar).

Nota-se na Figura 3 que a amostra 23, última classificada, apresentou o menor valor para o item transparência (Tabela 3S) e maior teor alcoólico (44,0\%) (Tabela 2S, Material Suplementar). A 
Amostra 23

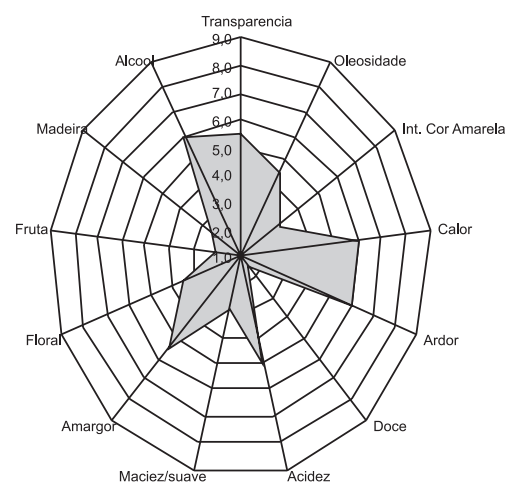

Amostra 8

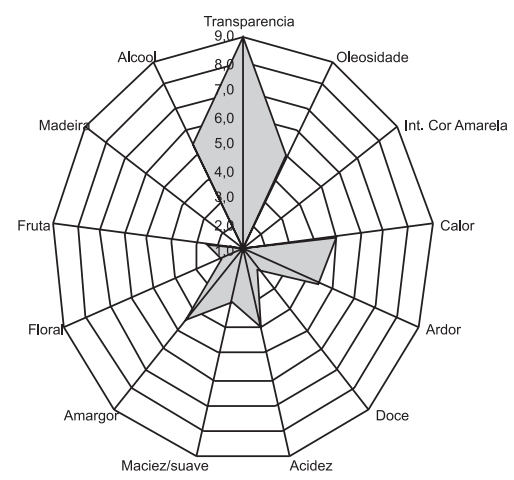

Figura 3. Perfil quantitativo (gráfico radar) das piores cachaças por parte da avaliação do painel de especialistas (exceto amostra 7)

amostra 7 apresentou o maior valor para dimetilsulfeto $\left(8,17 \mathrm{mg} \mathrm{L}^{-1}\right)$, entretanto, foi detectada nesta bebida a presença do corante caramelo $\left(5,00 \times 10^{-2} \mathrm{~g} \mathrm{~L}^{-1}\right)$ o qual pode ter mascarado aromas indesejáveis, permitindo assim a atribuição de um valor maior do nível hedônico, com respeito amostra de número 23.

Pode-se observar na Figura 4 que as amostras envelhecidas apresentaram teores inferiores de dimetilsulfeto com relação às amostras não envelhecidas. Este composto secundário é indesejável no aroma das aguardentes de cana, sendo frequentemente responsabilizado pelo aroma de repolho. ${ }^{27}$ Segundo a literatura, ${ }^{18}$ o baixo teor de dimetilsulfeto em cachaças envelhecidas pode ser explicado pela reação de oxidação, onde ocorre a formação de dimetilsulfóxido a partir do dimetilsulfeto. Certamente a elevada volatilidade do dimetilsulfeto (ponto de ebulição $37^{\circ} \mathrm{C}$ ) também deve contribuir para a redução dos teores deste composto ao longo do tempo de envelhecimento. ${ }^{26}$

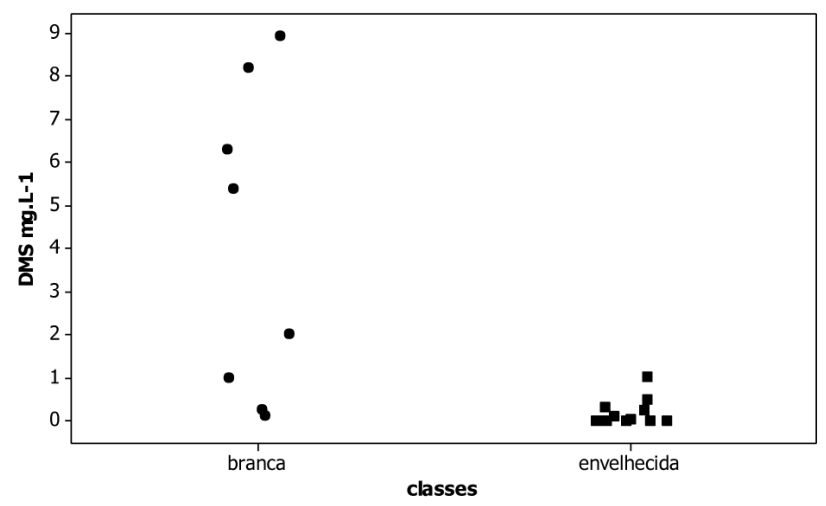

Figura 4. Correlação entre classes de cachaças e teor de dimetilsulfeto ( $\left.\mathrm{mg} \mathrm{L}^{-1}\right)$

Os dados obtidos por meio da análise sensorial (painel de especialistas) foram tratados utilizando-se PCA. Desta análise resultou a identificação de três grupos distintos (Figura 5) formados por amostras envelhecidas em carvalho, envelhecidas em jequitibá e não envelhecidas, As duas componentes do gráfico de scores apresentaram uma explicação do banco de dados original da ordem de $64,3 \%$. O conjunto de dados utilizado para a análise de $P C A$ foi elaborado com base nos valores da mediana de cada atributo avaliado na análise sensorial.

Observa-se na Figura 5 que os descritores sabor de madeira (PC1 $+\mathrm{PC} 2=0,70)$, intensidade de cor amarela $(\mathrm{PC} 1+\mathrm{PC} 2=0,65)$ e sabor de fruta $(\mathrm{PC} 1+\mathrm{PC} 2=0,60)$ foram os que mais contribuíram para a definição do grupo de cachaças envelhecidas em carvalho. Para as cachaças não envelhecidas, calor $(\mathrm{PC} 1+\mathrm{PC} 2=0,63)$ e ardor $(\mathrm{PC} 1$
$+\mathrm{PC} 2=0,56)$ foram os principais descritores para o agrupamento, seguidos pelos descritores amargor $(\mathrm{PC} 1+\mathrm{PC} 2=0,47)$, acidez $(\mathrm{PC} 1$ $+\mathrm{PC} 2=0,45)$ e sabor de álcool $(\mathrm{PC} 1+\mathrm{PC} 2=0,43)$. Já maciez/ suavidade $(\mathrm{PC} 1+\mathrm{PC} 2=0,51)$ e transparência $(\mathrm{PC} 1+\mathrm{PC} 2=0,37)$ foram os descritores que influenciaram no agrupamento das cachaças envelhecidas em jequitibá.

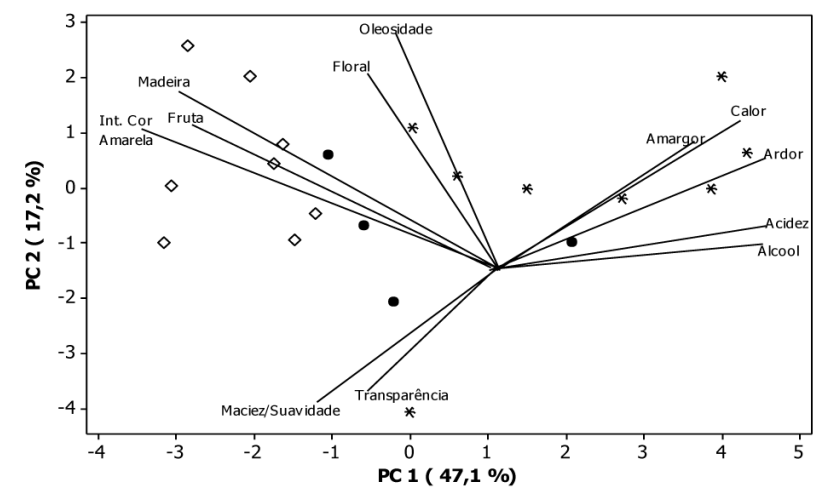

Figura 5. Gráfico de scores da PCA sobreposto ao gráfico de loading (* = cachaça não envelhecida; $\bullet$ = cachaça envelhecida em jequitibá; $\diamond=$ cachaça envelhecida em carvalho)

Com base no gráfico de loading, foram escolhidos os discriminantes de maior peso e posteriormente aplicou-se a análise de agrupamento hierárquico (HCA). O resultado dessa análise (Figura 6) sugere a formação de dois agrupamentos em cuja constituição estão discriminadores distintos. Um deles reúne as cachaças envelhecidas e o outro, as não envelhecidas com similaridade de 39,0\%. As amostras 8,17 e 22 foram classificadas como outliers.Estes resultados estão de acordo com os obtidos pela análise de componentes principais e pelo gerador hedônico.

Não foram observadas correlações entre os teores de carbamato de etila, cobre e ferro com o nível hedônico das cachaças.

Com relação ao método sensorial afetivo foram realizadas 1100 degustações, com uma média de 55 por amostra. O elevado número de provadores possibilitou uma avaliação mais segura da análise de preferência das cachaças, tornando-se evidente o grau de concordância e de repetibilidade nas análises.

Conforme os resultados, as amostras 5, 24 e 12 foram as que obtiveram maior índice de preferência global IGP (Figura 2S, Material Suplementar). Vale ressaltar que a amostra 24 foi a que apresentou o maior teor do corante caramelo $\left(3,40 \mathrm{~g} \mathrm{~L}^{-1}\right)$. 


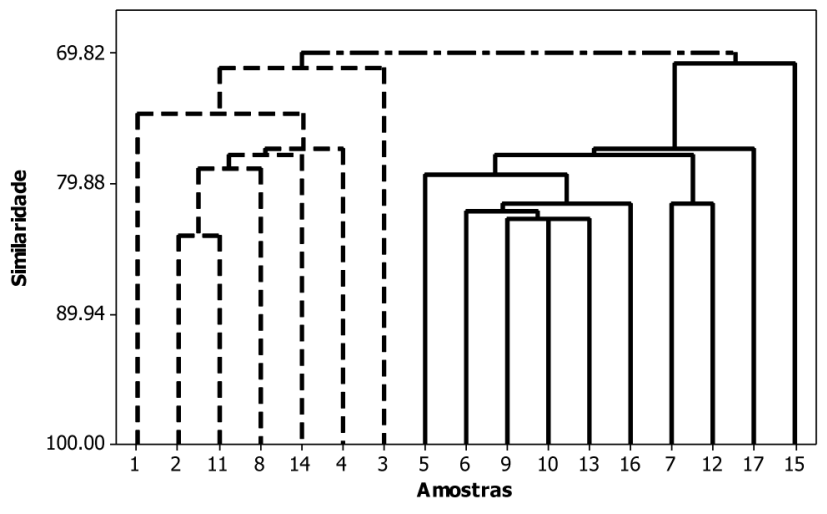

Figura 6. Dendrograma de similaridade entre cachaças não envelhecidas (...) e envelhecidas (-)

As amostras piores classificadas foram as de número 17 e 7 , e apresentaram como principal característica o não envelhecimento. Os índices de preferência determinados pelo painel de especialistas e o índice global de preferência obtido pelos consumidores são concordantes. As amostras que obtiveram a melhor classificação da parte dos consumidores se encontram entre aquelas que também foram bem avaliadas pelo painel de especialistas. A diferença entre os dois grupos está nos valores máximos dos índices, os quais foram maiores na avaliação dos consumidores.

Conhecer o nível de preferência do produto é importante, porém saber quais os caracteres que afetam positiva ou negativamente um produto é o ponto principal para melhorar as técnicas de processamento. Neste intuito foi desenvolvido um gerador hedônico para a cachaça (Figura 7), onde para a técnica estatística utilizada foram considerados os valores superiores a 0,30 , seja positivo ou negativo. Valores superiores a $-0,5$ e $+0,5$ demonstraram uma correlação significativa.

\section{Gerador Hedônico}

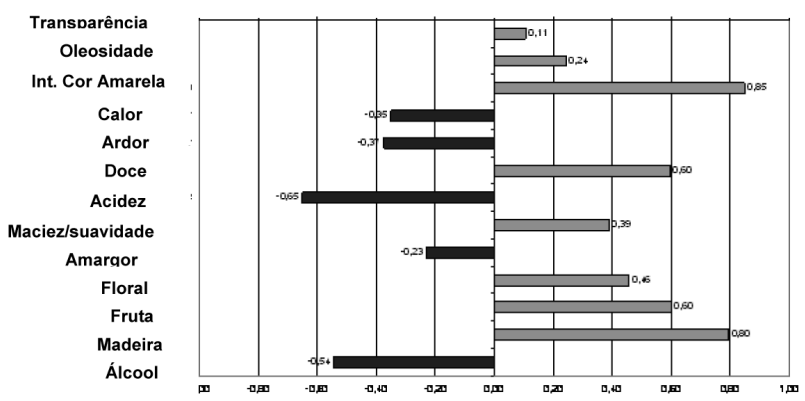

Figura 7. Correlação entre descrições objetivas de análise sensorial e índice global de preferência dos consumidores

A Figura 7 informa que o índice de preferência está principalmente correlacionado com a intensidade de cor amarela e com os sabores de madeira, doce e frutado. Existe uma correlação negativa com a acidez e o sabor de álcool, fatores estes que diminuem a preferência deste produto. De nosso conhecimento este é o primeiro gerador hedônico para a cachaça.

Este gerador hedônico vem confirmar uma pesquisa anterior para determinar o perfil do apreciador e do não consumidor da aguardente do estado de São Paulo realizada pelo Laboratório para o Desenvolvimento da Química da Aguardente (LDQA) envolvendo 1400 entrevistas em todo país. ${ }^{31}$

Nesta observou-se que a aguardente é escolhida principalmente pelo sabor e pela combinação sabor e teor alcoólico sendo preferidas as mais suaves para os apreciadores entrevistados. Os não consumidores relataram serem potenciais consumidores caso o sabor e o teor alcoólico da bebida fossem atenuados. A preferência pelo baixo teor alcoólico torna-se evidente no consumidor mais jovem, que prefere a bebida na forma de drinks. O consumidor sênior a ingere preferencialmente na forma pura, gelada ou on the rocks.

\section{CONCLUSÃO}

Os parâmetros objetivos e a definição do perfil qualitativo a serem avaliados sensorialmente em cachaça são: oleosidade, intensidade de cor amarela, calor, ardor, doce, acidez, maciez/suavidade, amargor, floral, frutado, madeira e álcool. Os resultados tanto do método descritivo como do método afetivo indicaram que as cachaças melhores classificadas foram as envelhecidas em barris de carvalho por um período não inferior a 24 meses. Em contrapartida, as piores classificadas apresentaram como principais características o não envelhecimento, elevada porcentagem alcoólica e elevado teor de dimetilsulfeto. Análises quimiométricas de $P C A$ e $H C A$ foram capazes de distinguir as cachaças analisadas por meio das variáveis sensoriais. Da análise de componentes principais $(P C A)$ resultou a identificação de três grupos distintos formados por amostras envelhecidas em carvalho, envelhecidas em jequitibá e não envelhecidas. Confirma-se, portanto, que o envelhecimento da cachaça é um importante parâmetro para a melhoria da sua qualidade sensorial, uma vez que esta etapa afeta a intensidade da cor amarela, o sabor de madeira, o sabor doce e o aroma de frutas, e diminui os aromas considerados negativos, como acidez, sabor de álcool e amargor. Com base nos resultados de sua análise foi possível elaborar um gerador hedônico para a cachaça.

\section{MATERIAL SUPLEMENTAR}

No material suplementar, disponível em http://quimicanova.sbq. org.br, na forma de arquivo PDF, com acesso livre, encontram-se os parâmetros tecnológicos das cachaças aprovadas para análise sensorial (Tabela 1S), bem como o resultado das análises químicas de teor alcoólico, cobre e ferro, carbamato de etila, dimetilsulfeto, caramelo (Tabela 2S), o perfil quantitativo das amostras analisadas pelo painel de especialistas (Tabela 3S), o índice hedônico das amostras por parte do painel de especialistas (Figura 1S) e o índice de global de preferência (IGP) realizado pelos consumidores (Figura $2 \mathrm{~S}$ ).

\section{AGRADECIMENTOS}

Ao CNPQ e à FAPESP pelo apoio financeiro.

\section{REFERÊNCIAS}

1. http://www.agrosoft.org.br/agropag/101414.htm, acessada em Setembro 2008.

2. Brasil, Ministério da Agricultura, Pecuária e Abastecimento; Decreto no 4062, de 21/12/2001; Diário Oficial da União 26/12/2001, seção 1, p. 4.

3. Boscolo, M.; Bezerra, C. W. B.; Cardoso, D. R.; Lima-Neto, B. S.; Franco, D. W.; J. Braz. Chem. Soc. 2000, 11, 86.

4. Nascimento, R. F.; Marques, J. C.; Keukeleire, D.; Lima-Neto, B. S.; Franco, D. W.; J. Chromatogr. 1997, 782, 13.

5. Aquino, F. W. B.; Boso, L. M.; Cardoso, D. R.; Franco, D. W.; Food Chem. 2008, 108, 784

6. Nascimento, E. S. P.; Cardoso, D. R.; Franco, D. W.; J. Agric. Food Chem. 2008, 56, 5488.

7. Cardoso, D. R.; Bettin, S. M.; Reche, R. V.; Lima-Neto, B. S.; Franco, D. W.; J. Food Comp. Anal. 2003, 16, 563. 
8. Bettin, S. M.; Isique, W. D.; Andersen, M. L.; Knudsen, S.; Skibsted, L. H.; Franco, D. W.; Eur. Food Res. Technol. 2002, 215, 169.

9. Galinaro, C. A.; Cardoso, D. R.; Franco, D. W.; J. Agric. Food Chem. 2007, 55, 3141.

10. Polastro, L. L. R.; Bosco, M.; Andrade-Sobrinho, L. G.; Lima-Neto, B. S.; Franco, D. W.; Ciênc. Tecnol. Aliment. 2001, 21, 78.

11. Isique, W. D.; Cardello, H. M. A. B.; Faria, J. B.; Ciênc. Tecnol. Aliment. 1998, 18, 356.

12. Pigott, J. R.; Distilled beverage flavour, VCA: Weinheim, 1989.

13. Cardoso, D. R.; Andrade-Sobrinho, L. G.; Leite-Neto, A. F.; Reche, R. V.; Isique, W. D.; Ferreira, M. M. C.; Lima-Neto, B. S.; Franco, D. W.; J. Agric. Food Chem. 2004, 52, 3429.

14. Brasil, Ministério da Agricultura, Pecuária e Abastecimento; Instrução Normativa ${ }^{\circ} 13$, de 29/06/2005; Diário Oficial da União 30/06/2005.

15. Muñoz, A. M.; Civille, G. V.; Carr, B. T.; Sensory evaluation in quality control, Van Nostrand Reinhold: New York, 1992.

16. Instituto Adolfo Lutz; Normas analíticas do Instituto Adolfo Lutz, $4^{\mathrm{a}} \mathrm{ed}$., IMESP: São Paulo, 2005.

17. Andrade-Sobrinho, L. G.; Boscolo, M.; Lima-Neto, B. S.; Franco, D. W.; Quim. Nova 2002, 25, 1074.

18. Cardoso, D. R.; Andrade-Sobrinho, L. G.; Lima-Neto, B. S.; Franco, D. W.; J. Braz. Chem. Soc. 2004, 15, 277.

19. Boscolo, M.; Andrade-Sobrinho, L. G.; Lima-Neto, B. S.; Franco, D. W.; Ferreira, M. M. C.; J. AOAC Int. 2002, 85, 744.
20. Associação Brasileira de Normas Técnicas; NBR 12994: Análise sensorial dos alimentos e bebidas, Rio de Janeiro, 1993.

21. Meilgaard, M.; Civille, G. V.; Carr, B. T. ; Sensory Evaluation Techniques, $3^{\text {rd }}$ ed., CRC Press: Boca Raton, 1999.

22. Odello, L.; Ugolini, A.; Violoni, M.; L`Assaggio: Analisi Sensoriale, I test descrittivi, Centro Studi Assagiatori: Brescia, 2007, vol. 14.

23. Odello, L.; L'Assaggio: Analisi Sensoriale, l'approccio facile, Brescia: Centro Studi Assagiatori: Brescia, 2004, vol. 10.

24. Odello, L.; Big Sensory Soft-2005; Programma di Statistica Applicata all'Analisi Sensoriale; Centro Centro Studi Assaggiatori: Brescia, 2005.

25. Minitab Statistical Software. Version 14; Minitab Inc, Pensilvânia, 2006.

26. Faria, J. B.; Cardello, H. M. A. B.; Boscolo, M.; Isique, W. D.; Odello, L.; Franco, D. W. ; Eur. Food Res. Technol. 2003, 218, 83.

27. Nascimento, R. F.; Cardoso, D. R.; Lima-Neto, B. S.; Franco, D. W.; Faria, J. B.; Quim. Nova 1998, 21, 735.

28. Litchev, V.; Am. J. Enol. Vitic. 1998, 40, 31.

29. Mosedale, J. R.; Puech, J. L.; Trends Food Sci. Tech. 1998, 9, 95.

30. Piggott, J. R.; Sharp, R.; Duncan, R. E. B.; The science and technology of whiskies, Longman Scientific \& Technical: New York, 1989.

31. Boscolo, M.; Bettin, S. M.; Bezerra, C. W. B.; Nascimento, R. F.; Cardoso, D. R.; Lima-Neto, B. S.; Franco, D. W.; trabalho não publicado. 


\section{AVALIAÇÃO SENSORIAL DE CACHAÇA}

Luigi Odello e Gian Paolo Braceschi

Centro Studi Assaggiatori, Galleria V.Veneto 9, 25128, Brescia, Itália

Fernanda Rosan Fortunato Seixas, Alexandre Ataide da Silva, Carlos Alexandre Galinaro e Douglas Wagner Franco*

Instituto de Química de São Carlos, Universidade de São Paulo, Av. do Trabalhador Sancarlense, 400, 13560-970 São Carlos - SP, Brasil

Tabela 1S. Parâmetros tecnológicos das amostras de cachaça aprovadas para a análise sensorial

\begin{tabular}{|c|c|c|c|c|}
\hline Código das amostras & Tipo de alambique & Envelhecida & Tipo de madeira do tonel & Tempo de envelhecimento (meses) \\
\hline 1 & Cobre & Sim & Carvalho & 24 \\
\hline 3 & Cobre & Sim & Carvalho & 36 \\
\hline 5 & Cobre & Sim & Carvalho & 24 \\
\hline 7 & Cobre & Não & - & 6 \\
\hline 8 & Cobre & Sim & Jequitibá & 24 \\
\hline 10 & Coluna/Inox & Não & - & 3 \\
\hline 11 & Cobre & Sim & Jequitibá & 36 \\
\hline 12 & Cobre & Sim & Carvalho & 24 \\
\hline 14 & Cobre & Não & - & 6 \\
\hline 15 & Cobre & Não & - & 6 \\
\hline 17 & Cobre & Sim & Jequitibá & 18 \\
\hline 19 & Cobre & Sim & Carvalho & 48 \\
\hline 20 & Cobre & Sim & Jequitibá & 36 \\
\hline 21 & Cobre & Não & - & 6 \\
\hline 22 & Cobre & Não & - & 11 \\
\hline 23 & Cobre & Não & - & 6 \\
\hline 24 & Cobre & Sim & Carvalho & 24 \\
\hline 28 & Cobre & Sim & Carvalho & 48 \\
\hline 33 & Cobre & Sim & Carvalho & 48 \\
\hline 35 & Cobre & Não & - & 6 \\
\hline
\end{tabular}

*e-mail: douglas@iqsc.usp.br 
Tabela 2S. Parâmetros químicos das amostras de cachaça aprovadas para a avaliação sensorial

\begin{tabular}{|c|c|c|c|c|c|c|}
\hline $\begin{array}{l}\text { Código das } \\
\text { amostras }\end{array}$ & $\begin{array}{l}\text { Teor alcoólico } \\
\qquad(\% \mathrm{v} / \mathrm{v})\end{array}$ & $\begin{array}{l}\text { Dimetilsulfeto }^{\mathrm{a}} \\
\quad\left(\mathrm{mg} \mathrm{L}^{-1}\right)\end{array}$ & $\begin{array}{l}\text { Cobre } \\
\left(\mathbf{m g ~ L} \mathbf{L}^{-1}\right)\end{array}$ & $\begin{array}{c}\text { Carbamato de Etila } \\
\qquad\left(\mathrm{mg} \mathrm{L}^{-1}\right)\end{array}$ & 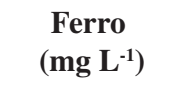 & $\begin{array}{l}\text { Caramelo } \\
\left(\mathrm{g} \mathrm{L}^{-1}\right)\end{array}$ \\
\hline 1 & 40 & $2,52 \times 10^{-1 b}$ & $1,30 \times 10^{-1 \mathrm{~b}}$ & $1,19 \times 10^{-1 b}$ & $7,10 \times 10^{-2 b}$ & $\mathrm{Nd}^{\mathrm{c}}$ \\
\hline 3 & 39 & $\mathrm{Nd}^{\mathrm{a}}$ & $3,20 \times 10^{-1 b}$ & $1,33 \times 10^{-1 b}$ & $5,70 \times 10^{-2 b}$ & $\mathrm{Nd}^{\mathrm{c}}$ \\
\hline 5 & 30 & $2,80 \times 10^{-2 b}$ & $1,70 \times 10^{-1 b}$ & $8,40 \times 10^{-2 b}$ & $5,90 \times 10^{-2} \mathrm{~b}$ & $6,00 \times 10^{-2 b}$ \\
\hline 7 & 40 & $8,17^{\text {b }}$ & $5,50 \times 10^{-2 b}$ & $9,60 \times 10^{-2 b}$ & $4,10 \times 10^{-2 b}$ & $5,00 \times 10^{-2 b}$ \\
\hline 8 & 38 & $9,00 \times 10^{-4 b}$ & $4,00 \times 10^{-1 b}$ & $7,30 \times 10^{-2 b}$ & $2,30 \times 10^{-2 b}$ & $\mathrm{Nd}^{\mathrm{c}}$ \\
\hline 10 & 36 & $6,28^{\mathrm{b}}$ & $6,00 \times 10^{-1 b}$ & $8,00 \times 10^{-2 b}$ & $1,60 \times 10^{-2 b}$ & $\mathrm{Nd}^{\mathrm{c}}$ \\
\hline 11 & 43 & $5,00 \times 10^{-1 b}$ & $4,60 \times 10^{-2 b}$ & $4,80 \times 10^{-2 b}$ & $1,40 \times 10^{-2 b}$ & $\mathrm{Nd}^{\mathrm{c}}$ \\
\hline 12 & 43 & $3,00 \times 10^{-3 b}$ & $1,50 \times 10^{-1 b}$ & $7,20 \times 10^{-2 b}$ & $2,20 \times 10^{-2 b}$ & $\mathrm{Nd}^{\mathrm{c}}$ \\
\hline 14 & 38 & $9,90 \times 10^{-1 \mathrm{~b}}$ & $1,50 \times 10^{-1 b}$ & $6,50 \times 10^{-2 b}$ & $6,00 \times 10^{-3 b}$ & $\mathrm{Nd}^{\mathrm{c}}$ \\
\hline 15 & 40 & $5,37^{\mathrm{b}}$ & $1,40 \times 10^{-1 b}$ & $6,90 \times 10^{-2 b}$ & $4,30 \times 10^{-2 b}$ & $\mathrm{Nd}^{\mathrm{c}}$ \\
\hline 17 & 33 & $1,01^{\mathrm{b}}$ & $1,20 \times 10^{-1 b}$ & $1,15 \times 10^{-1 b}$ & $1,70 \times 10^{-2 b}$ & $\mathrm{Nd}^{\mathrm{c}}$ \\
\hline 19 & 37 & $8,00 \times 10^{-4 b}$ & $5,10 \times 10^{-2 b}$ & $5,60 \times 10^{-2 b}$ & $5,00 \times 10^{-2 b}$ & $\mathrm{Nd}^{\mathrm{c}}$ \\
\hline 20 & 43 & $3,20 \times 10^{-1 b}$ & $8,00 \times 10^{-2 b}$ & $3,20 \times 10^{-2 b}$ & $2,80 \times 10^{-2 b}$ & $\mathrm{Nd}^{\mathrm{c}}$ \\
\hline 21 & 38 & $8,91^{\mathrm{b}}$ & $2,10 \times 10^{-1 b}$ & $3,80 \times 10^{-2 b}$ & $5,00 \times 10^{-3 b}$ & $\mathrm{Nd}^{\mathrm{c}}$ \\
\hline 22 & 41 & $1,98^{\mathrm{b}}$ & $5,40 \times 10^{-1 b}$ & $7,80 \times 10^{-2 b}$ & $7,00 \times 10^{-3 b}$ & $3,00^{\mathrm{b}}$ \\
\hline 23 & 44 & $2,45 \times 10^{-1 b}$ & $3,50 \times 10^{-1 b}$ & $8,00 \times 10^{-2 b}$ & $3,00 \times 10^{-2 b}$ & $\mathrm{Nd}^{\mathrm{c}}$ \\
\hline 24 & 42 & $1,62 \times 10^{-3 \mathrm{~b}}$ & $1,00 \times 10^{-1 b}$ & $1,08 \times 10^{-1 b}$ & $1,60 \times 10^{-2 b}$ & $3,40^{\mathrm{b}}$ \\
\hline 28 & 38 & $9,40 \times 10^{-2 b}$ & $5,40 \times 10^{-1 b}$ & $1,02 \times 10^{-1 b}$ & $2,50 \times 10^{-2 b}$ & $4,00 \times 10^{-2 b}$ \\
\hline 33 & 40 & $\mathrm{Nd}^{\mathrm{a}}$ & $7,80 \times 10^{-2} \mathrm{~b}$ & $8,10 \times 10^{-2 b}$ & $3,20 \times 10^{-2 b}$ & $1,30 \times 10^{-1 b}$ \\
\hline 35 & 41 & $9,20 \times 10^{-2 b}$ & $1,00 \times 10^{-2 b}$ & $9,30 \times 10^{-2 b}$ & $8,00 \times 10^{-4 b}$ & $\mathrm{Nd}^{\mathrm{c}}$ \\
\hline
\end{tabular}

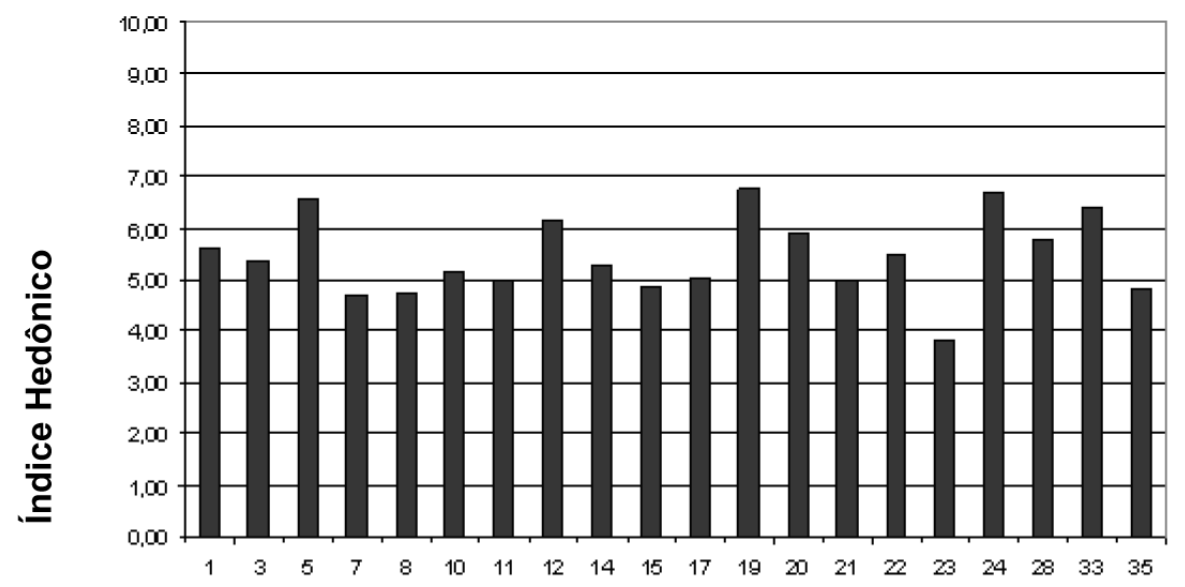

Amostras de Cachaças

Figura 1S. Índice hedônico do painel de especialistas (13 juízes) 
Tabela 3S. Perfil Quantitativo das amostras analisadas pelo painel de especialistas

\begin{tabular}{|c|c|c|c|c|c|c|c|c|c|c|c|c|c|}
\hline 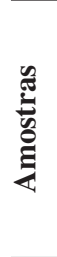 & 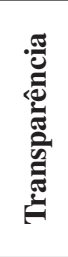 & $\begin{array}{l}\frac{0}{0} \\
\frac{0}{0} \\
\stackrel{0}{0} \\
0\end{array}$ & 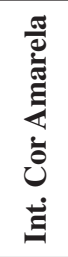 & $\frac{\grave{c}}{\tilde{J}}$ & $\stackrel{\dot{o}}{2}$ & $\stackrel{\mathscr{\circlearrowright}}{\stackrel{\Xi}{0}}$ & $\frac{\mathbb{d}}{\stackrel{0}{e}}$ & 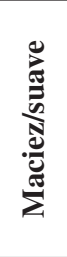 & 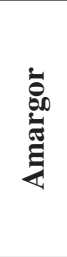 & $\overline{\overline{0}}$ & 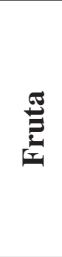 & $\frac{\sqrt[\pi]{\pi}}{\frac{\pi}{\pi}}$ & $\frac{\bar{O}}{8}$ \\
\hline 1 & 9,0 & 6,0 & 5,0 & 4,0 & 3,0 & 4,0 & 4,0 & 4,0 & 5,0 & 3,0 & 4,0 & 4,0 & 4,0 \\
\hline 3 & 9,0 & 4,0 & 6,0 & 4,0 & 3,5 & 3,0 & 4,0 & 4,0 & 3,0 & 3,5 & 3,0 & 3,5 & 4,0 \\
\hline 5 & 9,0 & 4,0 & 7,0 & 3,0 & 3,5 & 5,0 & 3,0 & 5,0 & 3,0 & 4,0 & 3,5 & 4,5 & 4,5 \\
\hline 7 & 9,0 & 4,0 & 1,0 & 6,5 & 6,0 & 2,5 & 5,5 & 3,0 & 6,0 & 3,0 & 3,0 & 1,0 & 6,5 \\
\hline 8 & 9,0 & 5,0 & 1,0 & 5,0 & 4,5 & 2,0 & 4,0 & 3,0 & 4,5 & 2,0 & 2,5 & 1,0 & 5,5 \\
\hline 10 & 9,0 & 3,0 & 1,0 & 4,0 & 4,0 & 4,5 & 3,5 & 5,5 & 4,0 & 2,0 & 2,5 & 1,0 & 4,0 \\
\hline 11 & 9,0 & 5,0 & 4,0 & 4,5 & 3,5 & 3,0 & 4,0 & 3,5 & 3,0 & 2,5 & 3,0 & 3,5 & 4,5 \\
\hline 12 & 9,0 & 5,0 & 5,0 & 4,0 & 4,5 & 3,5 & 4,0 & 4,0 & 2,5 & 3,0 & 3,0 & 4,0 & 4,0 \\
\hline 14 & 9,0 & 5,0 & 2,5 & 5,0 & 5,0 & 2,0 & 4,5 & 4,0 & 4,5 & 3,0 & 3,0 & 2,0 & 6,0 \\
\hline 15 & 9,0 & 5,0 & 1,5 & 5,5 & 5,5 & 3,0 & 5,0 & 4,0 & 4,5 & 3,0 & 2,0 & 1,5 & 6,0 \\
\hline 17 & 9,0 & 4,0 & 1,5 & 3,5 & 4,0 & 3,0 & 4,5 & 4,0 & 3,0 & 3,0 & 3,0 & 2,0 & 4,0 \\
\hline 19 & 9,0 & 5,0 & 8,0 & 4,0 & 3,5 & 3,0 & 3,5 & 5,0 & 3,0 & 3,5 & 4,0 & 4,5 & 4,0 \\
\hline 20 & 9,0 & $5, \mathrm{o}$ & 6,0 & 5,0 & 5,0 & 3,0 & 4,0 & 4,0 & 3,0 & 3,0 & 4,0 & 4,0 & 4,0 \\
\hline 21 & 8,0 & 5,0 & 2,0 & 5,0 & 4,5 & 3,5 & 4,0 & 5,0 & 4,0 & 4,5 & 2,5 & 2,0 & 5,5 \\
\hline 22 & 9,0 & 5,0 & 4,0 & 4,0 & 5,0 & 3,0 & 5,0 & 4,0 & 4,0 & 4,0 & 4,0 & 4,0 & 6,0 \\
\hline 23 & 5,5 & 4,5 & 3,0 & 6,0 & 6,0 & 1,5 & 5,0 & 3,0 & 5,5 & 3,5 & 2,0 & 2,5 & 6,0 \\
\hline 24 & 9,0 & 6,0 & 8,0 & 5,0 & 4,0 & 4,0 & 3,0 & 4,0 & 4,0 & 4,0 & 4,0 & 7,0 & 4,0 \\
\hline 28 & 9,0 & 5,0 & 6,0 & 4,0 & 4,0 & 3,0 & 4,0 & 4,0 & 4,0 & 3,0 & 4,0 & 5,0 & 4,0 \\
\hline 33 & 9,0 & 5,0 & 7,0 & 5,0 & 5,0 & 4,0 & 4,0 & 4,0 & 4,0 & 4,0 & 5,0 & 5,0 & 3,0 \\
\hline
\end{tabular}

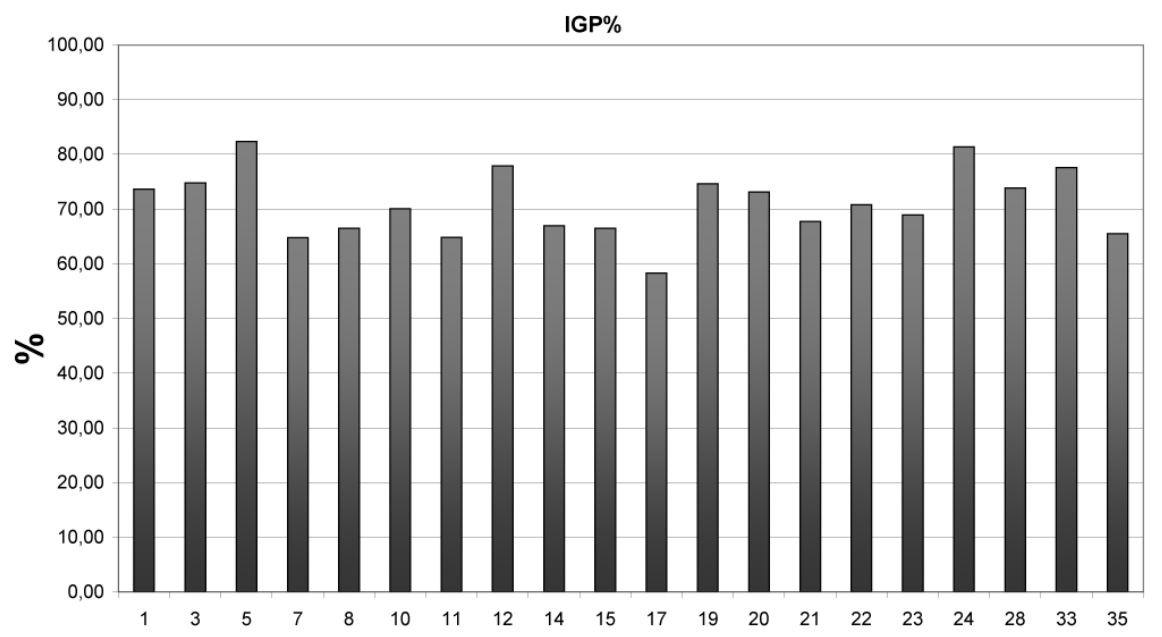

Amostras de Cachaça

Figura 2S. Índice global de preferência (IGP) do painel de consumidores 